\title{
Everyone wants a window seat
}

$\mathrm{T}$ hrough chance and unfathomable lengths of time, evolution has shown remarkable creativity, yet some creatures still defy imagination. The digenetic (requires more than one host) trematodes definitely fall into that category. Childhood imagination could not provide a more bizarre life cycle than that evolved by some of these helminths that bedevil so called higher creatures. Diplostomum spathaeum illustrates that phylum with an ophthalmic twist.

The life cycle of $D$ spathaeum begins as an egg in the faeces of a piscivorous bird, such as a gull or a pelican, as a definitive and unaffected host. It is in this host that the sexual phase occurs and the adult parasite lays its eggs in the bird's gastrointestinal tract. The excrement containing the eggs is deposited in a fresh water lake or stream. As the faeces drop to the floor of the lake or stream, perhaps on the northern Canadian shield where they are prevalent, the eggs embryonate for approximately 3 weeks and then hatch when exposed to light. The photonegative and short lived miracidia exit the eggs, find their way to a snail, often of the Lymnaea genus, and penetrate the flesh. In the snail, the miracidia penetrate the hepatopancreas and undergo metamorphosis into sporocysts which develop into larvae, called cercaria. Within approximately 40 days, the cercariae exit the molluscan host. The cercariae usually depart the snail when the water temperature is approximately $10^{\circ} \mathrm{C}$, assuring a summer discharge in temperate climates.

These free swimming parasites then seek any of several unfortunate species of fresh water fish, and are particularly fond of rainbow trout (illustrated on the upper left hand portion of this month's cover, without a cataract) and white suckers (illustrated on the lower left hand portion of the cover, with a cataract). Once their secondary intermediate host is located they penetrate the flank of the trout. Within the body of the trout, the parasite finds its way, unerringly, to the crystalline lens probably via nerves to the brain and then down the optic nerve or perhaps via the vascular system. The fluke, $D$ spathaceum, has almost complete site specificity for it requires the lens to complete its life cycle, although other members of this genus Diplostomum seem to require the eye, but not necessarily the lens.

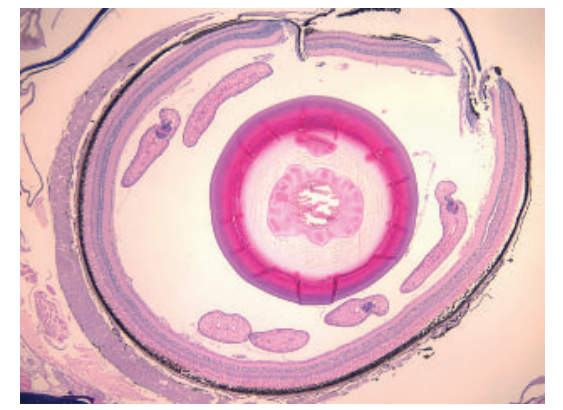

The image on the right of this month's cover is a fish eye containing Diplostomum species, but it cannot be confirmed as D spathaceum. The crystalline lens shows liquefaction and is cataractous and may represent a lenticular parasite that is not seen in the plane of this histological section.

For $D$ spathaceum, the crystalline lens is the principal target, and without those proteins the life cycle cannot be completed. Once the cercariae enter the lens, these mature into metacercariae in approximately 4 months at temperatures of approximately $12^{\circ} \mathrm{C}$. In the process of this maturation, a cataract forms. The right image on the cover reveals four cercariae in the vitreous and a forming cataract in a very young fish infected with this organism. Once cataractogenesis begins, it limits the fish's feeding, as trout are visual species and they will not take a fly they cannot see; thus this parasite may ruin valuable fisheries. As a fish becomes emaciated and is more heavily infected, it spends more time near the surface. The parasitised host will seek the light at the surface of the lake, where it is easy prey for predatory birds. Not only does the fish seek more light, but its protective visual mechanisms are obscured by its now forming cataract, making it crippled and easy prey for the attacking bird.

This platyhelminth requires its lenticular lunch for metamorphosis, and cannot mature without this diet. The chemical or mechanical attractant(s) the cercariae use to locate the eye is/ are not known or understood. Since our current understanding of ocular immunology includes the eye as privileged and the lens as encapsulated within that privilege, re-evaluation of that model might be necessary in the case of the association between fish and $D$ spathaceum. Normally, at least in humans, there appears to be no lens antigen release until the lens capsule is interrupted, and yet somehow this platyhelminth unerringly finds its way to these lens proteins to secure its meal of passage. Phototaxis may be a more likely mechanism although this would still present a puzzle as the parasite enters the flesh of the fish and must find its way to the lens.

$D$ spathaceum and related species parasitise a large variety of freshwater fish in North America and Europe-at least 105 species including important aquaculture species such as perch, roach, rainbow trout, bream, dace, gudgeon, and eel. Some authors report a female preponderance of infected individuals, although this may be an artefact of collection. Nevertheless, the benthic nature of the first intermediate host, the snail, and the proximity of the nesting female fish may put her at greater risk of infection.

This trematode (fluke) can and does infect humans although such infection is surprisingly rare. D spathaceum apparently is capable of penetrating the cornea and infecting the lens of frogs, turtles, birds, and even mammals although some experimental controversy exists. Interestingly, the cercariae can penetrate into, but not through, adult human corneas, and probably do not enter the anterior chamber in most instances. In some rabbits, corneal nebulae were produced by cercariae that did not penetrate. Perhaps these creatures are responsible for certain forms of nummular keratitis.

There are other piscine ocular parasites including the copepod parasites, Lernaeenicus sprattae and Phrixocephalus cincinnatus. Both of these can penetrate the eyes of various species and may destroy the eye or blind the fish although these have not so common or so bizarre a life cycle as $D$ spathaceum.

All of these parasites seem to jockey for a window seat.

\section{R Schwab \\ University of California, Davis, Sacramento, CA, USA; irschwab@ucdavis.edu}

Rainbow trout photograph (top left) by the author taken at the American River Trout Hatchery with thanks to Dennis A Redfern. Thanks to Jerold Thice, PhD, for his review of the essay. Sucker photograph (bottom left) by JD McLaughlin with thanks to Ron Hedrick and Thomas Waltzek for the pathological specimen. 